\title{
Study report on prevalence and risk factors associated with hepatitis B infection among dental health care workers
}

\author{
Ruchika K. Prasad ${ }^{1, *}$, Siva B ${ }^{2}$, Neeraj Kumar ${ }^{3}$, Vikram Panghal $^{4}$, Sangeeta Sunda ${ }^{5}$ \\ ${ }^{1,3,4}$ Dental Surgeon, ${ }^{2}$ Professor \& HOD, ${ }^{5}$ Reader, ${ }^{1}$ PP Lucknow, ${ }^{2}$ Dept. of Oral Medicine, Madhurai, ${ }^{3}$ Dept. of Prosthodontics, \\ Noida, Uttar Pradesh, ${ }^{4}$ Civil Hospital, Hisar, Haryana, ${ }^{5}$ Dept. of Orthodontics, Jan Nayak Ch. Devi Lal Dental College, Sirsa, \\ Haryana, India
}

*Corresponding Author:

Email: vikrampanghal1981@gmail.com

\begin{abstract}
Health care providers (HCP) are constantly working towards improving the health and healing patients to achieve this state of well-being. This is accomplished by a detailed clinical examination followed by the various investigative procedures and finally when a diagnosis is reached based on the clinical and investigative findings, a treatment plan suited to the needs of the patient is devised.

In providing these services to the patients, the HCP must be very careful and any carelessness on their part may expose to various diseases that the patient maybe suffering from.

This results in a vicious cycle of transmitting diseases from patients to HCP and auxiliaries and from them to other healthy patients. To stop this chain of transmission, the universal precaution that should always be followed is "all blood and hazardous body fluid must be considered infectious irrespective of a patient's diagnosis". ${ }^{1}$

The unintentional transmission by inoculation occurs more frequently than is recognized, however, is suggested by the viability of the agent or agents, the infectiousness of minute quantities of blood, the existence of detected carriers in the population, and the ever increasing use of injections. ${ }^{2}$

As mentioned, the other infectious disease of great importance other than HIV is Hepatitis. An inflammatory liver disease and is generally caused by a virus. Hepatitis implies injury to liver characterised by presence of inflammatory cells in the liver tissue. ${ }^{3}$

As a consequence of their parental mode of transmission and ability to establish chronic infection, hepatitis types HBV, HDV and HCV are of particular concern for oral health care professionals. ${ }^{4}$

$\mathrm{HB}$ is 100 times more infectious than HIV and HB is the second only to tobacco as a cause of major cancer in humans. ${ }^{5}$

This is a cross sectional study done in a dental college, in north India to check the prevalence of hepatitis $b$ in dental health care providers.
\end{abstract}

Keywords: Investigative, Treatment, Transmitting, Hazardous, Cancer.

\section{Introduction}

Hepatitis B virus (HBV) is the causative agent of one of the world's major infectious diseases with about 350 million people being chronic carriers of the virus. Hepatitis B infection is the 10th leading cause of death worldwide, as a significant number of the chronic carriers go on to develop liver cirrhosis or hepatocellular carcinoma (HCC) and over 1 million die annually from HBV associated liver disease. HCC is responsible alone for 320000 deaths per year. However, antiviral drugs are available for HBV infected individuals that may prevent the critical consequences of chronic liver disease, which emphasizes the significance of identifying infected individuals and monitoring the prevalence of the disease. Primary epidemiological data about HBV in any country would provide significant information to the program managers and health planers to control and manage the infection with reference to its etiological spectrum.

\section{Subjects and Methods}

A total of 499 dental health care professionals were participated in the screening for Hepatitis B Surface Antigen ( $\left.\mathrm{HB}_{S} \mathrm{AG}\right)$ of who were either studying or working in the institute. They were divided into four groups depending on their area of work. This study was approved by the Ethical Committee of Human and Animal Research of the college. The subjects for the study were categorized in four groups as follows:

1. Group I

2. Group II

3. Group III

4. Group IV

Group I: Faculty (Teaching technical staff): they compromised of 39 subjects $(7.8 \%)$

Group II: Student: all the undergraduate students, interns and post graduate students were included in this group; they compromised of the largest number of professionals participating in the present study. They were 357 (71.5\%). 
Group III: Non teaching technical staff (Hygienst and Technicians): they are denoted as allied in the results and they consisted of 70 individuals (14\%).

Group IV: Class IV employees and attendants: they comprised of subjects who did not directly deal with patient treatment but were exposed to patient's blood and other fluid like saliva. They were the sweepers and attendants and compromised of 33(6.6\%).

In all, extreme care was taken to selectively exclude the subjects mentioned below.

\section{Exclusion Criteria}

Subjects with recent history of:

1. Hepatitis B infection.

2. Any liver disease.

3. Pregnant women.

\section{Measures}

I. An informed consent was obtained by the health care professionals and a survey Performa was filled by them prior to the sample collection.

II. A questionnaire requesting demographic information about potential risk factors for HBV infection was also built and validated. This was a self-administered questionnaire, filled out by each participant in the presence of one researcher, at the same time of blood collection.

III. Materials for collection of serum sample:
1. A pair of sterile gloves
2. Disposable mouth mask
3. Tourniquet gauge disposable needle.
5. Sterile cotton
6. Vaccutainer $6 \mathrm{ml}$ plain
7. Remi R8C Centrifuge machine.
8. The ELISA analyser.
9. Reagent.

4. $5 \mathrm{ml}$ sterile disposable syringe with $23 \times 1$

\section{Methodology}

All the subjects were seated comfortably on chairs when the venous blood was drawn.

\section{Method of collection of blood and serum storage}

In each case $5 \mathrm{cc}$ of venous blood was drawn from the median cubital vein by using a sterile syringe and standard tourniquet as per the methodology of Howe G.L. for all the study subjects. The drawn blood was then transferred into dry, autoclaved test tubes and allowed to clot, followed by centrifugation at 3000 $\mathrm{rpm}$ for five minutes. The serum was stored in vacutainer at $2^{0}-8^{0} \mathrm{C}$ for not more than 24 hours. It was later subjected to serological estimation of Hepatitis B surface antigen in O.P. Chaudhary Hospital and Research Centre.

Throughout the procedure precautions were taken at every step to avoid haemolysis of blood which would render the sample unsuitable for analysis.
Method of estimation: ELISA, is the abbreviation of enzyme-linked immunosorbent assay.

\section{Principle}

Test principle: The basic principle of an ELISA is to use an enzyme to detect the binding of antigen (Ag) antibody (Ab). The enzyme converts a colourless substrate (chromogen) to a colored product, indicating the presence of Ag: Ab binding. An ELISA can be used to detect either the presence of Ags or Abs in a sample, depending on how the test is designed.

Procedure: For detecting antigens such as virus particles from a blood or faecal sample, the direct ELISA method is used. In this procedure the antigen is "trapped" between two layers of antibodies.

The results were an analysed and recorded accordingly.

\section{Specifying statistical procedure}

Continuous variable age was summarized as Mean \pm SD and compared by one way analysis of variance (ANOVA) followed by Newman-Keuls Post hoc test. Discrete (categorical) data (sex, marital status, were summarized in numbers (counts) and \%, and compared by $\chi^{2}$ test. A two-tailed probability $\mathrm{p}<0.05$ was considered statistically significant. Analyses were performed on SPSS (window version 15.0) while graphs were made on MS EXEL (MS Office 2007).

\section{Result}

The age of all 499 dental health professionals ranged from $18-54$ yrs with mean $( \pm$ SD) $24.84 \pm 5.84$ yrs. Among all dental health professionals, the proportions of males $(57.5 \%)$ were higher than females $(42.5 \%)$ and unmarried $(80.8 \%)$ were higher than married (19.2\%) as shown in table 1 . To find out which group of dental health professionals exposed the most and possible risk factors, all health professionals were sub-grouped into four groups (Faculty, Student, Allied and Class IV) according to area of work. The frequency distributions of subjects of four groups were summarized in Table 1. Among all dental health professionals, 7 (1.4\%) were Hepatitis B positive while 492 (98.6\%) were Hepatitis $B$ negative.

\section{Discussion}

HBV infection is an important public health problem all over the globe affecting both developed and developing countries. Health-care personnel (surgeons, pathologists, and other physicians, dentists, nursing staff, laboratory technicians, and blood bank personnel) have a higher incidence of hepatitis and prevalence of HBsAg or anti-HBs than those who have no occupational exposure to patients or blood products. ${ }^{7}$ 
In addition, the prevalence of HBV infection in dentists is about three times greater than that in the general population, which means that immunization of this high-risk group is extremely important. ${ }^{8}$

In the present study, 499 dental health care professionals were screened for the presence of hepatitis B surface antigen amongst whom 7 (1.4\%) tested positive who belonged to the class IV employees study group, which was similar to the prevalence rate of $6.0 \%$ found in Brazil by Enilza Maria Mendonça de Paiva et al (2008). ${ }^{2}$ This was significantly lower than studies conducted in the post vaccine era in South Brazil which had a prevalence of $31.2 \%$ in 250 professionals by Baldy et al (2003). In this way, the data disagrees with the assertive, "the dentists are considered three to five times more at risk for hepatitis B than the general population".

The age range varied from 18 years to 54 years the mean age being $24 \pm 5.84$ years. This was comparable to the mean age of 30 years reported by Saima Naz (2002) $)^{9}$ in a study conducted in India on health care professionals in Muzaffarabad. A study conducted on dental health care professionals in Brazil by Enilza Maria Mendonça de Paiva et al $(2008)^{2}$ reported the mean age was 37.2 years, and similar was the mean age of 38.55 years reported by Seyed-Moayed Alavian (2008). ${ }^{8}$ This was older than the mean age of our study group; hence our group consisted of younger dental professionals. Another aspect is that younger professionals adhere more closely to the standard guidelines resulting low prevalence of hepatitis B as seen in our study.

The mean age of the group who tested positive (class IV employees) for hepatitis B was 24.58 years (Std. Deviation= 6.12, Age STD Err $=1.07$, Age 95.00\% $=22.41$ and Age $+95.00 \%=26.75$ ) .

Our study compromised of 287 males $(57.5 \%)$ and 212 females $(42.5 \%)$ which was similar to the study conducted by Seyed-Moayed Alavian (2008). ${ }^{8}$ Also the study conducted by Saima naz (2002) ${ }^{9}$ concurred similar findings. This was in contrast to the study conducted by Mato Grosso do Sul et al $(2006)^{10}$ and Enilza Maria Mendonça de Paiva et al (2008). ${ }^{2}$

In this study it was observed that HBV infection rate predominated in the class IV employees. This pattern resembled the occurrence in the Malaysian community where infection was noted to be highest in the first and second decades of life by M Vadivale et al (1992). ${ }^{7}$

The increased risk among certain occupations have been attributed to the degree of exposure to blood and blood products and needle stick injuries rather than with patient handling as reported by $\mathrm{M}$ Vadivale et al (1992), ${ }^{7}$ Nelson et al ( 1987), Cottone (1985).

As compared to the class IV employees of our study group, the other three study gropus i.e. the staff, students and allied, all reported the prevalence to be $0 \%$. No other study reviewed by us has had sub groups within the study population and hence no studies have been quoted regarding this.

Recent data has suggested that the risk of HBV infection among HCWs has decreased with the availability of HBV vaccination (and the implementation of universal precautions). This reduced risk has not yet been fully assessed in hospital-based studies in developing countries this policy has not been vigorously adopted. A significant factor impeding universal implementation of $\mathrm{HBV}$ vaccination in HCWs is the cost of the vaccine, which is prohibitively expensive on a large scale. ${ }^{11}$

Healthcare workers and especially dentists can transmit hepatitis B infection to their patients or can be occupationally infected with HBV because of needle sticks or exposure to blood and other fluids.

The rate of hepatitis B vaccination varies greatly among dentists, and has not been accepted as a means of the possible elimination of the occupational risk of $\mathrm{HBV}$ infection.

The gaps of information found to be important but unavailable in this study was the duration of employment and speciality. The positive subject's family was not investigated, as it would further help us in knowing if the infection was acquired through the hospital or family.

With this we conclude that educational efforts must go beyond the mere teaching of universal precautions and should include the introduction of safer products and clinical procedures that can minimize the risks associated with the hands-on learning. Availability of HBV vaccination facilities for dental healthcare workers and education of dentists on cross infection control, along with encouraging them to use protective barrier methods, may overcome the risk of infection transmission.

Hence, all prospective entrants to dental school must be screened for hepatitis B, hepatitis C, HIV and TB. The requirements, should also apply to entrants for dental hygiene and dental therapy courses also, stating that admission will be conditional on the results of this testing for blood-borne viruses (BBVs).

In conclusion, with the rationale that most carriers of HBV are either free of symptoms or have diseases that have not yet been diagnosed, we recommend strict compliance with universal precautions. Consequently, the hospital/ college must ensure the materials for primary precautions which are appropriate to the level, of the tasks, are always available to HCWs. We have also provided a scientific basis for recommending a mandatory vaccination of our doctors and dentists with HBV vaccine. The vaccination should be offered free of charge to the HCWs. 
In order to improve staff screening and vaccination, health services need to invest more resources in this aspect of occupational health and safety. Explicit directions about how to implement the policy and consistency across organisations would be beneficial as currently there is variation in implementation between facilities, which contributes to the problems.

An electronic database for storing this information, which can be shared across healthcare facilities, is also required. This would prevent duplication of screening processes and assist the mobile workforce in the healthcare system.

To compromise on this is to place lives at risk, and to lower morale among both the HCWs and the public.

\section{References}

1. Enilza Maria Mendonça de Paival. Anaclara Ferreira Veiga Tiplle; Eliane de Paiva e Silva; Divina das Dores de Paula Cardoso; Serological Markers And Risk Factors Related To Hepatitis B Virus In Dentists In The Central West Region Of Brazil; Braz J Microbiol 2008;39:251-6.

2. Margie Patlak, Drs. Baruch Blumberg, Maurice Hilleman, and William Rutter beyond Discovery: The Path from Research to Human Benefit, a project of the National Academy of Sciences. February 2000

3. W.H.O./CDS/EPR/LYO/2006.1,Communicable Disease Surveillance and Response Systems, a guide to planning. Department of Communicable Diseases Surveillance and Response, 2002.

4. (Late) R. Ananthnarayna PhD, CK Jayaram Panikar MD, Ananthnarayan And Paniker's Textbook Of Microbiology: $7^{\text {th }}$ Edition, Orient Longman, p:547-55

5. Martin S Greenberg, DDS, Micheal Glick, DMD, Burkets Oral Medicine, Diagnosis And Treatment, $10^{\text {th }}$ Edition, 2008, B C Decker, p:534-6

6. Jagdev Heir, And Vincent B. Ziccardi; Transmission of Infectious Disease in the Dental Setting; Spec Care Dent 65:378-82.

7. M. Valdivale, T.C.Tan and C.N Ong Sero-Prevalence of Hepatitis Infection among Dental Professionals: Singapore Med J 1992;33:367-9.

8. Seyed-Moayed Alavian, Morteza Izadi, Ali-Akbar Zare, Maryam Moghani Lankarani, Shervin Assari, Mohammad-Mahdi Vardi. Survey Of The Level Of Anti-Hbs Antibody Titer In Vaccinated Iranian General Dentists; Spec Care Dent 2008;28(6):265-70.

9. Saima Naz, Mushtaq Ahmad And Humayun Asghar. Prevalence of Hepatitis 'B' Among Hospital Personnel in Combined Military Hospital (CMH) Muzaffarabad. Int J Agric Biol 1560-8530/2002/04-2-227-230.

10. Mato Grosso do Sul et al; Seropositivity for Hepatitis B Virus, Vaccination Coverage, And Vaccine Response In Dentists From Campo Grande, Brazil; Mem Inst Oswaldo Cruz, Rio de Janeiro, 2006;101(3):263-7.

11. Shepard et al Hepatitis B and C: Prevalence and risk factors associated with seropositivity among children in Karachi, Pakistan. BMC Infect Dis 2006,6:101. 